\title{
Multiple mating by females of two Bactrocera species (Diptera: Tephritidae: Dacinae)
}

\author{
A. Chinajariyawong1, R.A.I. Drew ${ }^{2 *}$, A. Meats ${ }^{3}$, \\ S. Balagawi ${ }^{2}$ and S. Vijaysegaran ${ }^{2}$
}

\author{
${ }^{1}$ Institute of Agriculture Technology, Walailak University, Thasala, \\ Nakhon Si Thammarat, 80161, Thailand: ${ }^{2}$ International Centre for the \\ Management of Pest Fruit Flies, Griffith School of Environment, Griffith \\ University, 170 Kessels Rd, Nathan QLD 4111, Australia: ${ }^{3}$ School of \\ Biological Sciences A12, University of Sydney, NSW 2006, Australia
}

\begin{abstract}
Multiple mating was investigated in two tephritid species when females were under minimal male pressure because they were each confined with a single male in cages $20 \times 20 \times 20 \mathrm{~cm}$ and observed daily until they died. Laboratory-reared females of Bactrocera cucumis (French) lived up to 274 days and refractory periods averaged 59-63 days. However, the distribution of matings among B. cucumis females was not significantly different to that expected by chance. Wild females of Bactrocera cacuminata (Hering) reared from field-collected fruits of Solanum mauritianum Scopoli lived for up to 134 days and mated up to three times with refractory periods between matings averaging 27-39 days. The distribution of the number of matings among females of $B$. cacuminata was non-random because of the high proportion of non-maters (50\%); but, when only females mating more than once were considered, there was no significant departure from random expectation.
\end{abstract}

Keywords: refractory period, Bactrocera cacuminata, Bactrocera cucumis, SIT

(Accepted 30 July 2009)

\section{Introduction}

Multiple mating, especially polyandry, is common in tephritids and refractory periods for females have been reported in the range of one day to four weeks, whereas males are generally found to be able to mate daily for several days or maintain the ability to mate for several weeks (Tychsen \& Fletcher, 1971; Fletcher \& Giannakakis, 1973; Fay \& Meats, 1983; Prokopy \& Roitberg, 1984; Robacker et al., 1985; Kuba \& Itô, 1993; Landolt, 1994; Meats \& Fay, 2000; Kraaijeveld et al., 2005; Radhakrishnan \& Taylor, 2007, 2008).

*Author for correspondence

Fax: + 61737353697

E-mail: d.drew@griffith.edu.au
The fitness consequences of multiple mating have been extensively reviewed (Ridley, 1988; Birkhead \& Møller, 1998; Arnqvist \& Nilsson, 2000). For tephritid fruit flies, it is possible that repeated mating can shorten the lifespan of females, but this may be offset by increased fecundity (Chapman et al., 1998).

Much research on multiple mating of tephritid females has focussed on the means by which male ejaculate inhibits remating during the refractory period (Fletcher \& Giannakakis 1973; Kuba \& Itô, 1993; Mossinson \& Yuval, 2003; Kraaijeveld \& Chapman, 2004; Harmer et al., 2006; Radhakrishnan \& Taylor, 2007, 2008). Other factors that affect the length of the refractory period and remating frequency include density, biased sex ratio and long-term adaptation to crowded culture conditions (Robinson et al., 2002; Vera et al., 2002; Kraaijeveld et al., 2005). Notwithstanding the fact that many tephritids 
in the field can form mating aggregations (Tychsen, 1977; Sivinski, 1984; Arita \& Kaneshiro, 1989; Prokopy et al., 1996), the high densities in some laboratory cultures can be as artificial as those used in the technique of 'forced mating' that is used to produce unusual events, such as interspecific hybrids (Takai et al., 1984; Taylor \& Craig, 1985; Ye et al., 1992; Pike \& Meats, 2002). Thus, different laboratory protocols can yield different results on multiple mating, even for the same species. It would be desirable to use conditions of density and sex ratio similar to those found in the field; and, indeed, multiple mating can be detected in the field by finding DNA microsatellite evidence for polyandry (Tripet et al., 2003; Kraaijeveld et al., 2005; Song et al., 2007). However, this does not give precise evidence of the numbers of matings per female and cannot provide any on refractory period.

This paper describes research that used conditions that reduced any potential for forced mating to a minimum because flies were caged in male-female pairs in $20 \times 20 \times 20 \mathrm{~cm}$ cages, and each pair was observed daily during the normal dusk mating period until the female died. Thus, polyandry was not possible unless a male that died was replaced. Two species studied were Bactrocera cucumis (French) and Bactrocera cacuminata (Hering). The former is a serious pest of commercial cucurbits in Queensland; whereas the latter is widespread along the entire Australian eastern seaboard; and, although it is not a pest, it has some potential to be one because it can be cultured in a variety of commercial fruits (Fitt, 1986; Drew, 1989).

\section{Materials and methods}

\section{Study insects}

B. cucumis were obtained from a laboratory colony approximately ten generations old and held at the ICMPFF laboratories, Griffith University, Brisbane. Laboratory colonies were maintained accord to the methods of Swaine et al. (1978), with about 6-8 weeks elapsing between generations. $B$. cacuminata were reared from infested fruits of its major host plant, Solanum mauritianum Scopoli, that were collected from the field at Mt Gravatt, Brisbane.

All flies were reared, and the experiments conducted, under constant environmental conditions of $25^{\circ} \mathrm{C}\left( \pm 1^{\circ} \mathrm{C}\right)$, $60-70 \%$ relative humidity and a photoperiod of $9 \mathrm{~h}: 15 \mathrm{~h}$, light: dark cycle with a natural dusk from a window occurring at the start of the dark phase. Under these conditions, fly mating activity occurred under dusk illumination between 1730 to 1930 hours. Flies for the experiments were allowed to emerge from puparia within $30 \times 30 \times 30 \mathrm{~cm}$ stock cages, enclosed with a fine synthetic mesh, immediately separated into sexes in separate cages and then provided with a diet of sugar, yeast protein (MP Biomedicals LLC enzymatic yeast hydrolysate) and water.

\section{Mating study}

For B. cucumis, mating observations were conducted on 62 pairs of virgin flies combined at three days after emergence from puparia. Each pair was confined in a cage $(20 \times 20 \times 30 \mathrm{~cm})$ and provided with the same diet as in the stock cages of emerged males and females noted above. Over time, males that died were replaced with males from the same cohort that had also been held with a single female under the same environmental conditions. Females that died were not replaced. Each pair of flies was monitored daily during the dusk phase and records made of mating pairs. These observations were conducted using a dull red torch light in order not to disturb mating pairs. Observations continued for 274 days. It was not possible to systematically record courtship activity because of the number of pairs and the poor light.

For B. cacuminata, two sets of observations were conducted along similar lines to those above. In the first case, 22-day-old virgin males and females were combined in 35 single pairs in separate cages. Observations continued until the last female died at 134 days. In the second case, all procedures were identical except that 45 pairs of virgin flies were set up when the flies were seven days old.

\section{Statistical analyses}

Statistical analyses established the significance $(P)$ and predictive power (percentage of variance explained, $100 r^{2}$ ) of various correlations. These correlations were: (i) between the number of matings and age of death; (ii) the delay between the first and second mating, and the second and third mating; (iii) the delay between the first and second, and the third and fourth mating; (iv) the delay between the second and third, and the third and fourth mating; and (v) the delay between the age of first mating and each of the aforementioned delays. Significance was defined in relation to a critical $P$ of $\alpha=0.05$ that was subjected to Dunn-Sidâk's adjustment to $\alpha^{\prime}$, where $\alpha^{\prime}=1-(1-\alpha)^{1 / n}$ and $n=$ the number of comparisons in the analysed group (Sokal \& Rohlf, 1995).

Comparison was also made between the distribution of number of matings per female and that expected by chance from the binomial distribution. When a fly can mate no more than once per fortnight, the binomial probability of mating $r$ times in $n$ fortnights is:

$$
W r_{(i)}=n ! /[r(i) !(n-r(i)) !] \cdot p^{r(i)} q^{n-r(i)}
$$

where $r(i)$ is a number from zero to $n$ inclusive, $q=1-p$, and $\Sigma W_{r(i)}=1$.

The number of flies expected to mate $0,1 \ldots n$ times was found by multiplying the pertinent value of $W_{r}$ by the number of flies starting a trial.

\section{Results}

\section{Survival and mating in B. cucumis}

Sixty of the 62 pairs of B. cucumis that were caged together at three days old mated at least once and, of these 60,53 pairs $(88 \%)$ mated twice, 31 pairs $(52 \%)$ mated three times, 11 pairs (18\%) mated four times and 2 pairs (3\%) mated five times. The mean refractory period between matings was remarkably constant but the standard deviations were wide; the means $( \pm S E)$ in days were, respectively, $59 \pm 40.4,60.6 \pm 29.4$ and $63.0 \pm 17.0$. This was associated with a wide asynchrony in mating times with a great deal of variation between pairs in terms of the number of matings achieved by a given age (fig. 1).

B. cucumis females lived up to 274 days (mean $171 \pm 7.5$ $\mathrm{SE}$. There was a significant relationship between the number of matings and the time of female death $(P=0.005 ; n=58)$, indicating that the longer-lived flies had mated more often. The correlation explained $13 \%$ of the variance. 


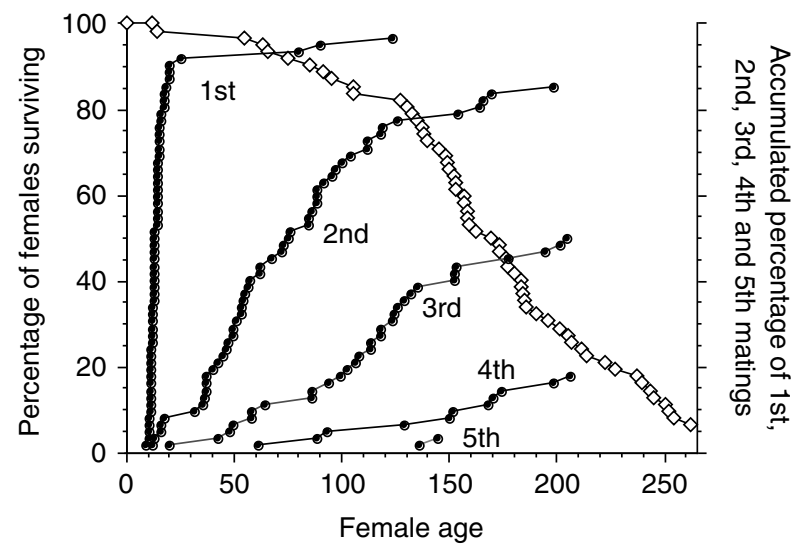

Fig. 1. Percentage of female B. cucumis (white symbols) surviving and (black symbols) accumulated percentages of first, second and subsequent matings.

\section{Relation of periods between successive matings in B. cucumis}

The times (refractory periods) between the first and second matings in B. cucumis and the times between the second and third matings were significantly correlated if treated as a single comparison but not in terms of the Dunn-Sidâk adjusted level of significance $\left(\alpha^{\prime}\right)$ for multiple comparisons, $\left(P=0.04, \alpha^{\prime}=0.017 ; n=31\right)$. The correlation accounted for $14 \%$ of the variance. The times between the first and second, and the third and fourth matings were not correlated $(P=$ $0.64 ; n=11$, accounting for $2.4 \%$ of variance) and neither were the times between the second and third, and the third and fourth mating $(P=0.36 ; n=31$, accounting for $9.4 \%$ of the variance).

There was no correlation between age of first mating in $B$. cucumis and the time between the first and second mating or the third and fourth mating $(P=0.46 ; n=53$ and 0.56 ; $n=31$, respectively).

\section{Survival and mating in B. cacuminata}

For B. cacuminata, 23 of the 35 pairs caged together at 22 days old mated once and, of these 23,17 pairs $(74 \%)$ mated twice and 5 pairs $(22 \%)$ mated three times. Similarly, 27 of the 45 pairs caged together at seven days old mated once and of these 27, 15 pairs (56\%) mated twice and 4 pairs $(15 \%)$ mated three times. The mean refractory periods were lower than the values found for B. cucumis, being about 27-39 days with SEs ranging from 6.3-14.6.

B. cacuminata females caged together at 22 days old lived for a maximum of 134 days (mean 92 days $\pm 4.5 \mathrm{SE}$ ) and those caged together at seven days lived a maximum of 130 days (mean 86 days \pm 5.2 SE). There was no significant difference between these two means $(t=0.84, \mathrm{df}=78, P$ (twotailed $)=0.4$ ). The correlation between lifespan and the number of matings in $B$. cacuminata that mated was significant $(P=<0.001, n=50)$, indicating that the longer-lived flies tended to mate more often, and this relationship accounted for $40 \%$ of the variance.

The correlations between the time of first mating in $B$. cacuminata and the time between the first and second matings, and second and third were not significant $(P=0.95$; $n=32$ and $P=0.65 ; n=9$, respectively). Likewise, the
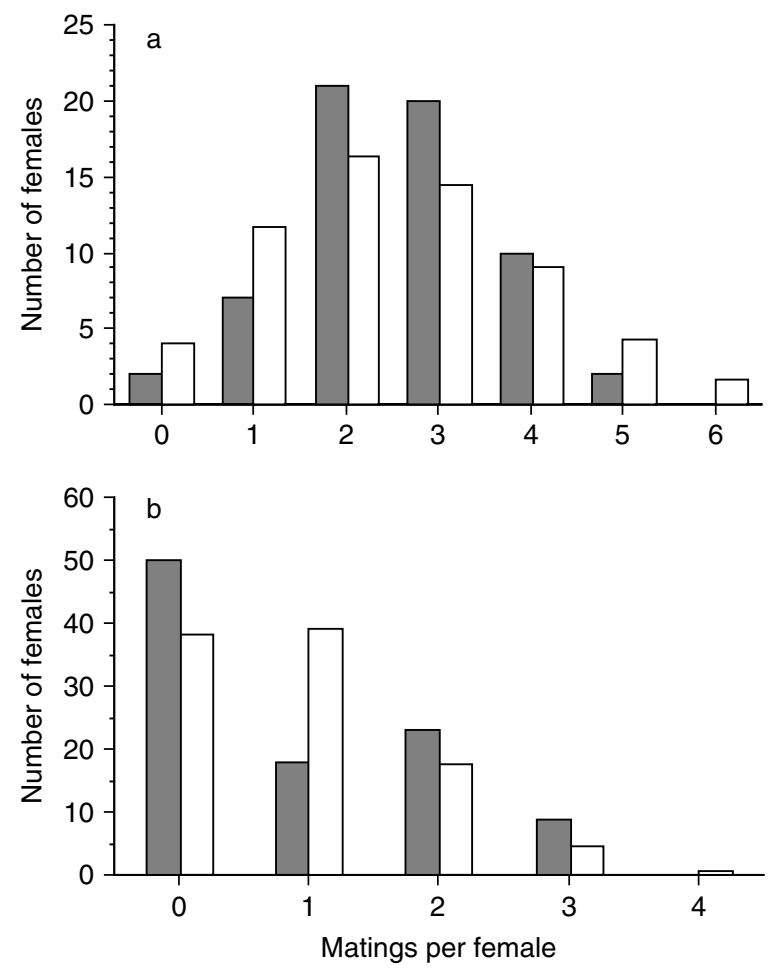

Fig. 2. The distribution of frequency of matings per female in (a) B. cucumis and (b) B. cacuminata compared to the binomial expectation ( $\square$, observed; $\square$, binominal expectation).

correlation between the time between the first and second matings, and the second and third was not significant $(P=0.66 ; n=9)$.

\section{Mating, chance and the expectation from the binomial model}

For B. cucumis, the relationship between the distribution of matings per female and the binomial expectation is shown in fig. 2a. There were 62 B. cucumis females in the study, and a total of 159 matings were recorded in the 20 fortnights of observation; thus, the binomial probability of observing a mating by a given female in a given fortnight was 159/20/ $62=0.1282$. The distribution of matings per female expected over the whole period according to this probability and the number of periods is compared to the observed frequencies in fig. 2. The two distributions do not deviate significantly $\left(\chi^{2}=9.21, P=0.101, \mathrm{df}=5\right)$.

For B. cacuminata, the relationship between the distribution of matings per female and the binomial expectation is shown in fig. $2 \mathrm{~b}$. The binomial probability for this species (following the procedure outlined above) was calculated as $91 / 8 / 100=0.11375$. In this case, the two distributions are significantly different $\left(\chi^{2}=21.1, P<0.001, \mathrm{df}=3\right)$. Because the result could have been influenced by the large proportion of non-maters, a comparison was made using only the numbers of B. cacuminata females that mated more than once. In this case, 18 females mated once only (thus, mated zero times more than once), 23 mated once more than once and 9, and zero mated twice and three times more that once, respectively. The binomial probability (deducting a fortnight for the first mating period) was calculated as $41 / 7 / 50=0.1171$. 
The binomial expectations using this probability did not differ significantly from the observed values $\left(\chi^{2}=3.56, P=\right.$ 0.313, df=3).

\section{Discussion \\ Multiple mating and survival}

A high percentage of females of both B. cacuminata and B. cucumis mated at least twice and in all experiments. The refractory period for $B$. cacuminata exceeded that recorded for B. tryoni by Fletcher \& Giannakakis (1973) while that for B. cucumis was twice as long. The demonstrated multiple mating in monogamous pairs would suggest a potential for polyandry in the field. This has been borne out for one of our study species by Song et al. (2007) who found, through molecular examination of the progeny of wild $B$. cacuminata females, that they mated with at least two males. The higher number of matings per female in B. cucumis was associated with their long life spans. The maximum was 274 days compared to 134 days for B. cacuminata in this study and 140 days recorded in laboratory conditions for $B$. tryoni by both Drew et al. (1983) and Meats (2006). The difference in the degree of multiple mating between B. cucumis and B. cacuminata is most likely due to the greater opportunity for mating in the former afforded by its longer lifespan. Within species, female insects generally gain fitness benefits from mating frequently (generally polyandrously) but this may not be manifested in increased longevity (Arnqvist \& Nilsson, 2000; Ivy \& Sakaluk, 2005; Gershman, 2007; Seslija et al., 2008).

\section{The role of chance in individual pairings}

The very weak or non-significant relationships between the intervals between matings in individual B. cucumis mating pairs are consistent with the distribution of matings among pairs being not significantly different to that expected by chance. However, the distribution of the number of matings among females in B. cacuminata was non-random, despite non-significant relationships between the intervals between matings. In this case, the cause appears to be the unexpectedly high proportion of non-maters (50\%) because, when the distribution of mating frequencies of females mating more than once are considered, there is no significant departure from random expectation.

The importance of null models (such as the Poisson) to the analysis of mating data has been recognised by several authors because what appears to be governed by sexual selection or other biotic influences may also be explained as the outcome of random processes (Sutherland, 1985; Bradbury \& Andersson,1987; Hartley \& Shepherd, 1995; Friedl \& Klump, 2005; Snyder \& Gowaty, 2007). Meats \& Fay (2000) established that matings per male in B. tryoni were consistent with a random model, whereas Focardi \& Tinelli (1996) found that a model of behaviour in leks governed by random processes not only produced results usually consistent with random expectation but occasionally produced results that were not.

\section{The SIT and female refractory periods}

The SIT, or the control of a pest by the mass release of conspecific sterile males, relies on the ability of the sterile males to mate with wild females and cause sterility of any eggs and, failing that, to impose as long a refractory period as possible. However, remating frequency may be increased by crowding, biased sex ratio and long-term adaptation to crowded laboratory culture (Robinson et al., 2002; Vera et al., 2002; Kraaijeveld et al., 2005). Thus, adaptation to mass rearing can decrease the refractory period of females, although whether this is solely due to a deficiency in sterile males is not certain. For example, Radhakrishnan \& Taylor (2007) using B. tryoni from an SIT factory found about $15 \%$ of females remated within one day rather than the period of four weeks reported by Fletcher \& Giannakakis (1973). Kraaijeveld et al. (2005) considered that, under such circumstances, polyandry could be a drawback in genetic control programmes. However, it would make no difference to the ratio of sterile males to wild males required as long as the ejaculate of released sterile males was as effective in imposing a refractory period as wild males (Curtis, 1985). An effective sterilizing dose of radiation not only causes dominant lethal mutations in spermatids and spermatozoa (fatal to any fertilized egg) but also prevents the initiation of further spermatogenesis. Thus, depending upon the timing of irradiation, a virgin sterile male will either have no sperm or a limited exhaustible supply (Ashburner, 1989). For two Bactrocera species examined to date, it is not necessary for sperm to be present in the ejaculate in order for mating to cause a refractory period (Kuba \& Itô, 1993; Harmer et al., 2006; Radhakrishnan \& Taylor, 2007, 2008). If the ejaculate of the sterile males does not cause such a long female refractory period as that of wild males, this would have to be rectified by releasing sterile males at a higher rate.

When small production areas of about $1 \mathrm{~km}$ across have to be treated with the SIT (e.g. see Barnes, 2007), polyandrous multiple mating of wild females could be advantageous, as it would result in the remating of any that invaded from neighbouring untreated sites, and such females would stand a very high probability of mating with sterile males (Helsinki et al., 2008).

\section{References}

Arita, L.H. \& Kaneshiro, K.Y. (1989) Sexual selection and lek behavior in the Mediterranean fruit fly Ceratitis capitata (Diptera: Tephritidae). Pacific Science 43, 135-143.

Arnqvist, G. \& Nilsson, T. (2000) The evolution of polyandry: multiple mating and female fitness in insects. Animal Behaviour 60, 145-164.

Ashburner, M. (1989) Drosophila. A laboratory Handbook. 1331 pp. Cold Spring Harbor, New York, USA, Cold Spring Harbor Laboratory Press.

Barnes, B.N. (2007) Privatizing the SIT: a conflict between business and technology? pp. 449-456 in Vreysen, M.J.B., Robinson, A.S. \& Hendrichs, J. (Eds) Area-Wide Control of Insect Pests. Dordrecht, The Netherlands, Springer.

Birkhead, T.R. \& Møller, A.P. (1998) Sperm Competition and Sexual Selection. 826 pp. London UK, Academic Press.

Bradbury, J.W. \& Andersson, M.B. (Eds) (1987) Sexual Selection: Testing of Alternatives. 308 pp. Chichester, UK, John Wiley.

Chapman, T., Miyatake, T., Smith, H.K. \& Partridge, L. (1998) Interactions of mating, egg production and death rates in Mediterranean fruit fly, Ceratitis capitata females. Proceedings of the Royal Society of London, Series B 265, 1879-1894.

Curtis, C.F. (1985) Genetic control of insect pests: growth industry or lead balloon? Biological Journal of the Linnaean Society 26, 359-374. 
Drew, R.A.I. (1989) The tropical fruit flies (Diptera: Tephritidae: Dacinae) of the Australasian and Oceanian regions. Memoirs of the Queensland Museum 26, 1-521.

Drew, R.A.I., Courtice, A.C. \& Teakle, D.S. (1983) Bacteria as a natural source of food for adult fruit flies (Diptera: Tephritidae). Oecologia 60, 274-284.

Fay, H.A.C. \& Meats, A. (1983) The influence of age, ambient temperature, thermal history and mating history on mating frequency in males of the Queensland fruit fly, Dacus tryoni. Entomologia Experimentalis et applicata 34, 273-276.

Fitt, G.P. (1986) The roles of adult and larval specialisations in limiting the occurrence of five species of Dacus (Diptera: Tephritidae) in cultivated fruits. Oecologia 69, 101-109.

Fletcher, B.S. \& Giannakakis, A. (1973) Factors limiting the response of the females of the Queensland fruit fly, Dacus tryoni, to the sex pheromone of the male. Journal of Insect Physiology 19, 1147-1155.

Focardi, S. \& Tinelli, A. (1996) May random processes explain mating success in leks? Behavioural Processes 36, 227-237.

Friedl, T.W.P. \& Klump, G.M. (2005) Sexual selection in the lek-breeding European tree frog: body size, chorus attendance, random mating and good genes. Animal Behaviour 70, 1141-1154.

Gershman, S.N. (2007) Female Gryllus vocalis field crickets gain diminishing returns from increasing numbers of matings. Ethology 113, 1099-1106.

Harmer, A.M.T., Radhakrishnan, P. \& Taylor, P.W. (2006) Remating inhibition in female Queensland fruit flies: Effects and correlates of sperm storage. Journal of Insect Physiology 52, 179-186.

Hartley, I.R. \& Shepherd, M.A. (1995) Random female settlement model can explain polygyny in the Corn Bunting. Animal Behaviour 49, 1111-1118.

Helsinki, M.E.H., Hood, R.C. \& Knols, B.G.J. (2008) A stable isotope dual-labelling approach to detect multiple insemination in un-irradiated and irradiated Anopheles arabiensis mosquitoes. Parasitic Vectors 1, 1-9.

Ivy, T.M. \& Sakaluk, S.K. (2005) Polyandry promotes enhanced offspring survival in decorated crickets. Evolution 59, 152159.

Kraaijeveld, K. \& Chapman, T. (2004) Effects of male sterility on female remating in the Mediterranean fruitfly, Ceratitis capitata. Proceedings of the Royal Society of London, Series B (Suppl.) 271, S209-S211.

Kraaijeveld, K., Katsoyannos, B.I., Stavrinides, M., Kouloussis, N.A. \& Chapman, T. (2005) Remating in wild females of the Mediterranean fruit fly, Ceratitis capitata. Animal Behaviour 69, 771-776.

Kuba, H. \& Itô, Y. (1993) Remating inhibition in the melon fly, Bactrocera (Dacus) cucurbitae (Diptera, Tephritidae) - copulation with spermless males inhibits female remating. Journal of Ethology 11, 23-28.

Landolt, P.J. (1994) Mating frequency of the papaya fruit fly (Diptera: Tephritidae) with and without host fruit. Florida Entomologist 77, 305-312.

Meats, A. \& Fay, H.A.C. (2000) Distribution of mating frequency among males of the Queensland fruit fly, Bactrocera tryoni, in relation to temperature, acclimation and chance. General $\mathcal{E}$ Applied Entomology 9, 27-30.

Meats, A. (2006) Attributes pertinent to over-wintering potential do not explain why Bactrocera neohumeralis (Hardy) (Diptera: Tephritidae) does not spread further south within the geographical range of B. tryoni (Froggatt). Australian Journal of Entomology 45, 20-25.

Mossinson, S. \& Yuval, B. (2003) Regulation of sexual receptivity of female Mediterranean fruit flies: old hypotheses revisited and a new synthesis proposed. Journal of Insect Physiology 49, 561-567.

Pike, N. \& Meats, A. (2002) The potential for mating between Bactrocera tryoni (Froggatt) and B. neohumeralis (Hardy) (Diptera: Tephritidae). Australian Journal of Entomology 41, 70-74.

Prokopy, R.J. \& Roitberg, B.D. (1984) Foraging behavior of true fruit flies. American Scientist 72, 41-49.

Prokopy, R.J., Poramarcom, R., Sutantawong, M., Dokmaihom, R. \& Hendrichs, J. (1996) Localization of mating behavior of released Bactrocera dorsalis flies on host fruit in an orchard. Journal of Insect Behavior 9, 133-142.

Radhakrishnan, P. \& Taylor, P.W. (2007) Seminal fluids mediate sexual inhibition and short copula duration in mated female Queensland fruit flies. Journal of Insect Physiology 53, 741-745.

Radhakrishnan, P. \& Taylor, P.W. (2008) Ability of male Queensland fruit flies to inhibit receptivity in multiple mates, and the associated recovery of accessory glands. Journal of Insect Physiology 54, 421-428.

Ridley, M. (1988) Mating frequency and fecundity in insects. Biological Reviews 63, 509-549.

Robacker, D.C., Ingle, S.J. \& Hart, W.G. (1985) Mating frequency and response to male-produced pheromone by virgin and mated females of the Mexican fruit fly. The Southwestern Entomologist 10, 215-221.

Robinson, A.S., Cayol, J.P. \& Hendrichs, J. (2002) Recent findings on Medfly sexual behavior: Implications for SIT. Florida Entomologist 85, 171-180.

Seslija, D., Marecko, I. \& Tucic, N. (2008) Sexual selection and senescence: do seed beetle males (Acanthoscelides obtectus, Bruchidae, Coleoptera) shape the longevity of their mates? Journal of Zoological Systematics and Evolutionary Research 46, 323-330.

Sivinski, J. (1984) Effect of sexual experience on male mating success in a lek forming tephritid Anastrepha suspensa. Florida Entomologist 67, 126-130.

Snyder, B.F. \& Gowaty, P.A. (2007) A reappraisal of Bateman's classic study of intrasexual selection. Evolution 61, 24572468.

Sokal, R.R. \& Rohlf, F.J. (1995) Biometry: The Principles and Practice of Statistics in Biological Research. 3rd edn. 887 pp. New York, USA, WH Freeman and Co.

Song, S.D., Drew, R.A.I. \& Hughes, J.M. (2007) Multiple paternity in a natural population of a wild tobacco fly, Bactrocera cacuminata (Diptera: Tephritidae), assessed by microsatellite DNA markers. Molecular Ecology 16, 23532361.

Sutherland, W.J. (1985) Chance can induce a sex difference in variance in mating success and explain Bateman's data. Animal Behaviour 33, 1341-1352.

Swaine, G., Corcoran, R.J. \& Davey, M. (1978) Commodity treatment against infestations of the cucumber fly Dacus (Austrodacus) cucumis French, in cucumbers. Queensland Journal of Agricultural and Animal Sciences 35, 5-9.

Takai, K., Kanda, T., Oguma, Y., Cheong, W.H., Joesoef, A.M. \& Sucharit, S. (1984) Postmating reproductive isolation between 7 members of the Anopheles hyrcanus species group in East Asia. Japanese Journal of Sanitary Zoology 35, 251-259 
Taylor, D.B. \& Craig, G.B. (1985) Unidirectional reproductive incompatibility between Aedes berlandi and Aedes hendersoni (Diptera: Culicidae) Annals of the Entomological Society of America 78, 769-774.

Tripet, F., Touré, Y.T., Dolo, G. \& Lanzaro, G.C. (2003) Frequency of multiple inseminations in field-collected Anopheles gambiae females revealed by DNA analysis of transferred sperm. American Journal of Tropical Medicine and Hygiene 68, 1-5.

Tychsen, P.H. \& Fletcher, B.S. (1971) Studies on the rhythm of mating in the Queensland fruit fly, Dacus tryoni. Journal of Insect Physiology 17, 2139-2156.
Tychsen, P.H. (1977) Mating behaviour of the Queensland fruit fly, Dacus tryoni (Diptera: Tephritidae) in field cages. Journal Australian Entomological Society 16, 459465.

Vera, M.T., Wood, R.J., Cladera, J.L. \& Gilburn, A.S. (2002) Factors affecting female remating frequency in the Mediterranean fruit fly (Diptera: Tephritidae). Florida Entomologist 85, 156-164.

Ye, Y., Xu, Z., Huang, R. \& Zhao, X. (1992). Hybridization experiments using Anopheles minimus from Guangxi and Yunnan. Chinese Journal of Parasitology \& Parasitic Diseases 10, 287-289. 